\title{
Introduction : Réflexions sur la construction des identités dans des régions frontalières
}

Doris Wastl-Walter et Ulrike H. Meinhof

Traducteur : Michel Deshaies

(2) OpenEdition

Journals

Édition électronique

URL : http://journals.openedition.org/rge/2209

DOI : $10.4000 /$ rge.2209

ISSN : $2108-6478$

Éditeur

Association des géographes de l'Est

Édition imprimée

Date de publication : 1 septembre 2003

ISSN : 0035-3213

Référence électronique

Doris Wastl-Walter et Ulrike H. Meinhof, «Introduction : Réflexions sur la construction des identités dans des régions frontalières », Revue Géographique de l'Est [En ligne], vol. 43 / 4 | 2003, mis en ligne le 19 novembre 2010, consulté le 24 septembre 2020. URL : http://journals.openedition.org/rge/2209 ; DOI : https://doi.org/10.4000/rge.2209

Ce document a été généré automatiquement le 24 septembre 2020.

Tous droits réservés 


\title{
Introduction : Réflexions sur la construction des identités dans des régions frontalières
}

\author{
Doris Wastl-Walter et Ulrike H. Meinhof
}

Traduction : Michel Deshaies

1 Le 1er mai 2004, 59 ans après la fin de la seconde guerre mondiale, 10 nouveaux pays (dont 8 issus de l'ancien bloc soviétique ou de l'ancienne Yougoslavie) sont entrés dans l'Union européenne. Ainsi, d'une fédération d'états occidentaux, l'Union européenne s'est transformée en une "nouvelle Europe ». Avec l'entrée dans l'Union, l'une des frontières les plus disputées entre l'Est et l'Ouest est devenue une frontière interne entre partenaires. Le fer est devenu de la soie. Quinze ans auparavant, le rapprochement entre les anciens états ennemis s'était déjà esquissé avec la disparition de la frontière intérieure allemande qui, jusqu'à la chute du mur de Berlin, représentait le symbole des différences idéologiques, économiques et sociales et des conflits entre les régimes de l'est et de l'ouest. Avec l'engagement de négociations pour l'incorporation d'états situés plus à l'est, la vision de la communauté européenne se rapproche de plus en plus de la conception culturelle et géographique traditionnelle d'un continent européen aux frontières allant jusqu'à l'Asie et l'Afrique et peut-être même au-delà avec l'éventuelle entrée de la Turquie.

2 Le point de départ de notre travail a été l'hypothèse que les frontières ne sont pas naturelles, comme si elles étaient voulues par dieu, mais le résultat de négociations politiques et de rapports de force et que, comme ceux-ci, elles peuvent changer. De plus, nous avons aussi considéré que l'identité n'est pas quelque chose qui est donné et fixé une fois pour toutes, mais le produit d'un discours et d'une construction sociale, et en conséquence elle peut aussi changer. Nous nous sommes particulièrement attachés au jeu changeant des frontières politiques et des identités spatiales, ainsi qu'à leur interaction.

3 Notre objectif était, à travers l'optique de la frontière (" the lens of the border ", voir Berg et van Houtum, eds, 2003), d'étudier les processus qui, dans le discours, 
conduisent à se délimiter du voisin politique. Aussi, la frontière de l'ancien rideau de fer nous a paru le mieux convenir. Elle a séparé des régions qui, pendant longtemps, étaient unies à la fois sur le plan politique et fonctionnel et qui étaient même très fortement intégrées. Or, au cours des 60 à 70 dernières années, une période sur laquelle les habitantes et les habitants les plus âgés peuvent encore avoir une vue globale, cet espace frontalier a connu un sort changeant: du système frontalier pratiquement infranchissable (sauf au sud) à la frontière politique réglementée et perméable, puis à la frontière de Schengen et depuis le 1er mai 2004, une frontière interne à l'Union européenne.

4 Nous voulions savoir comment les gens de la frontière avaient transposé ces changements dans leurs représentations d'eux-mêmes et de l'étranger, comment ils se conceptualisaient eux-mêmes et leurs voisins de l'autre côté de la frontière et quels étaient les points communs ou les différences qu'ils percevaient.

5 Le numéro thématique proposé ici est le résultat des recherches d'une équipe de chercheurs qui, entre 2000 et 2003, ont étudié des lieux séparés ou situés à proximité, des deux côtés de cette frontière, depuis le nord avec les villes germano-polonaises de Guben/Gubin et Görlitz/Zgorzelec jusqu'aux villes frontières italiano-slovènes de Gorizia/Nova Gorica. On a également incorporé dans nos recherches des lieux situés le long de l'ancienne frontière intérieure allemande, des deux côtés de la Saale, en Thuringe et en Bavière (Haute Franconie). Ce travail a été rendu possible par des fonds de recherche européens issus du cinquième programme cadre de la commission européenne, si bien que l'on a pu non seulement mener des recherches sur des lieux situés à une frontière nationale particulière, mais aussi quasiment de la Mer baltique à la Mer méditerranée, dans une perspective comparative. Nous nous sommes posés la question de savoir si, et de quelle manière, les bouleversements des structures politiques, idéologiques et économiques des différents États se reflétaient dans les expériences et les identités de familles qui, pendant trois générations, avaient passé toute leur vie ou la plus grande partie de celle-ci dans ces lieux. A cela étaient liées toutes les questions sur le maintien ou au contraire la disparition de l'hostilité à travers les différentes générations. Dans quelle mesure les possibilités de créer une identité transnationale dans une Europe unie était-elle acceptée ou refusée par les habitants de la frontière ? Dans quelle mesure les expériences personnelles et collectives, si typiques dans ces communes, d'hostilité, de séparation, d'expulsion, de fuite, de réinstallation, de marginalisation et d'autres effets de la guerre ainsi que de la guerre froide, se prolongent-elles jusque dans le présent ? Dans quelle mesure sont-elles accentuées ou atténuées par les conditions culturelles, économiques, sociales et politiques du présent ? Comment et pourquoi les stéréotypes négatifs existant sur les voisins de l'autre côté de cette frontière se renforcent-ils? Dans quelle mesure, où et dans quelles conditions $\mathrm{y}$-a-t-il un rapprochement de part et d'autre de la frontière ou de l'ancienne frontière ? Ces questions étudiées par notre équipe de recherche ne sont que partiellement traitées dans ce numéro thématique (voir les différents articles dans Meinhof éditeur (2002 et 2003), et pour des indications bibliographiques supplémentaires sur chaque article voir le site http://www.borderidentities.com).

Contrairement aux études quantitatives à très grande échelle réalisées sur les questions identitaires et sociales en Europe (voir par exemple le « Eurobarometer » et le « Social Attitudinal Survey» de la fondation européenne pour la science), notre équipe a travaillé suivant une méthode issue de l'ethnographie et de l'analyse du discours qui 
accorde beaucoup d'importance à la formulation thématique et linguistique des expériences vécues. Le contexte dans lequel a été effectuée la collecte des récits personnels et des relations de la vie des différents membres de la famille a consisté en conversations détendues et non structurées, à deux et parfois même à trois, le plus souvent dans diverses pièces d'habitation de nos interlocuteurs de différentes générations. Il nous a paru particulièrement important, d'une part d'influencer le moins possible les représentations thématiques, d'autre part de conserver les possibilités de comparaison des conclusions. La solution à cette quadrature du cercle méthodologique a été fournie par une méthode employée dans une étude antérieure de Meinhof et Galasinski, dans laquelle ce sont moins des questions que des images spécifiques d'une époque et d'un lieu qui constituaient le support des conversations (Meinhof \& Galasinski 2000). Pour chacun de nos lieux, nous avions une série de photographies correspondant aux trois époques importantes de nos générations (1930-1945 ; 1946-1989/90 ; actuellement), choisies en fonction de critères communs :

1. les images devaient représenter des endroits (ponts, bâtiments, monuments, etc.) dans nos lieux qui seraient immédiatement reconnaissables par chaque habitant ;

2. les images devaient pouvoir être replacées dans le temps par les générations qui ne connaissaient cette époque qu'à travers des récits ;

3. les images devaient représenter ou se signaler par des aspects émotionnels et symboliques dans l'existence des communes.

7 Ainsi, nous avons choisi par exemple comme support pour les trois générations vivant à l'ancienne frontière intérieure allemande, des images de ponts sur la rivière Saale qui pouvaient être clairement associées à chacune des époques par les habitants : ainsi, par exemple a) l'image d'un pont en pierre sur la Saale qui, jusqu'en 1945, reliait le village franconien de Tiefengrün et l'ancien moulin de Kühn du côté ouest de la rivière avec la ville thuringienne de Hirschberg et sa tannerie situées du côté est ; b) les restes du pont (le seul vestige du pont détruit par les SS en 1945) sur les deux rives de la Saale qui allait ensuite, entre 1945 et 1949, séparer les zones d'occupation américaine et soviétique, puis à partir de 1949, les deux états allemands; et c) l'étroite passerelle piétonnière en bois, ouverte en décembre 1989 , et le nouveau pont autoroutier après la réunification: mais de toute façon sans la tannerie qui ne pouvait pas survivre aux conditions économiques et écologiques nouvelles de l'Allemagne réunifiée. Des images de cette sorte ont suscité des réactions spontanées de nos informateurs qui, en aucune manière, ne sont restés captifs du cadre spatio-temporel tracé par les images, mais ont débouché sur des récits et des arguments avec lesquels on pouvait construire des interprétations signifiantes. En dehors de la relative liberté de choix des thèmes et de leur contenu argumentaire, la méthode du support iconographique permettait en même temps une plus grande liberté de choix de la conversation. On a laissé à nos interlocuteurs le choix de nommer et d'exprimer des lieux et des noms de rues (les désignations nouvelles en polonais ou anciennes en allemand, en slovène ou en italien), des états (RDA/ ancienne RDA, zone est, nouveaux Länder, Allemagne de l'Est, etc.). En regardant ensemble les images, nous avons ainsi évité d'introduire nous-mêmes de telles désignations, si riches de significations et de sens politique pour les représentations identitaires de nos interlocuteurs. Mais dans le même temps, les images ont permis de construire une structure grossièrement comparable pour tous les interviewers. 
8 Les textes ainsi rassemblés forment une collection unique de matériel et ont permis une analyse comparative des constructions identitaires et des expériences vécues par trois générations de familles le long de cette longue frontière. Les différents articles et extraits rassemblés dans ce volume sont essentiellement basés sur ce travail en commun. Dans le numéro de la revue, ils sont ordonnés du nord vers le sud et chacun étudie un thème particulièrement significatif ou caractéristique de son espace.

Dans la première contribution, Heidi Armbruster et Ulrike Meinhof caractérisent les trois phases de constitution de la frontière, qui sont aussi trois phases de changement de la mentalité frontalière : la fin des années 50 , au cours desquelles les habitants de la frontière s'habituent lentement à la coupure de leur espace vécu; les années 60 pendant lesquelles la guerre froide définit le statu-quo et par là même la délimitation mentale de la frontière des deux côtés, et enfin la période postérieure à 1989-90 qui certes supprime la frontière, mais ne crée aucune nouvelle solidarité. L'article discute de la façon dont ces différentes phases sont évoquées et représentées dans les interviews. Les aspects concrets des paysages comme leur incarnation y jouent un rôle particulier puisque la frontière a toujours été double: un mur physiquement infranchissable et un biais socio-culturel. L'article montre comment l'imbrication des dimensions physique et culturelle a façonné l'expérience historique et l'image que se fait d'elle-même la population frontalière.

Aleksandra Galasinska s'est intéressée aux villes voisines de Görlitz et Zgorzelec séparées par la Neisse/ Nysa. Le centre historique est resté en Allemagne et a été récemment rénové, mais c'est la ville polonaise qui a connu le développement le plus important au cours des dernières décennies. Dans cet article, on étudie la coopération entre les deux villes, d'une part comme discours tenu par les élites et les fonctionnaires qui a conduit entre autres à la fondation de l'Euro-cité Görlitz-Zgorzelec en mai 1998. D'autre part, il y a aussi la pratique quotidienne de toutes les citoyennes et de tous les citoyens des deux villes qui se révèle bien souvent être très loin d'être coopérative. Les résultats des recherches montrent l'existence d'un fossé entre la politique et la pratique quotidienne à l'échelle locale et finalement peu de liens entre ces deux plans. Dans cette contribution, on montre que les problèmes démographiques, sociaux, culturels et économiques des deux villes pourraient être mieux résolus par une coopération transfrontalière et qu'ainsi tous profiteraient du projet «Euro-cité ». On est cependant encore très éloigné de la constitution d'une telle communauté et de telles identités transnationales.

Ilona Scherm, Pavla Tiserová étudient les deux communes de Bärenstein (Allemagne) et Vejprty (République tchèque) qui depuis toujours constituent une unité séparée par le ruisseau frontalier Pöhlbach/Polava. Au cours du siècle passé, cette frontière se faisait sentir plus ou moins fortement; cependant, de 1946 à 1990, elle était devenue pratiquement infranchissable. 
Figure 1 : L'ancien Rideau de fer et localisation des zones étudiées

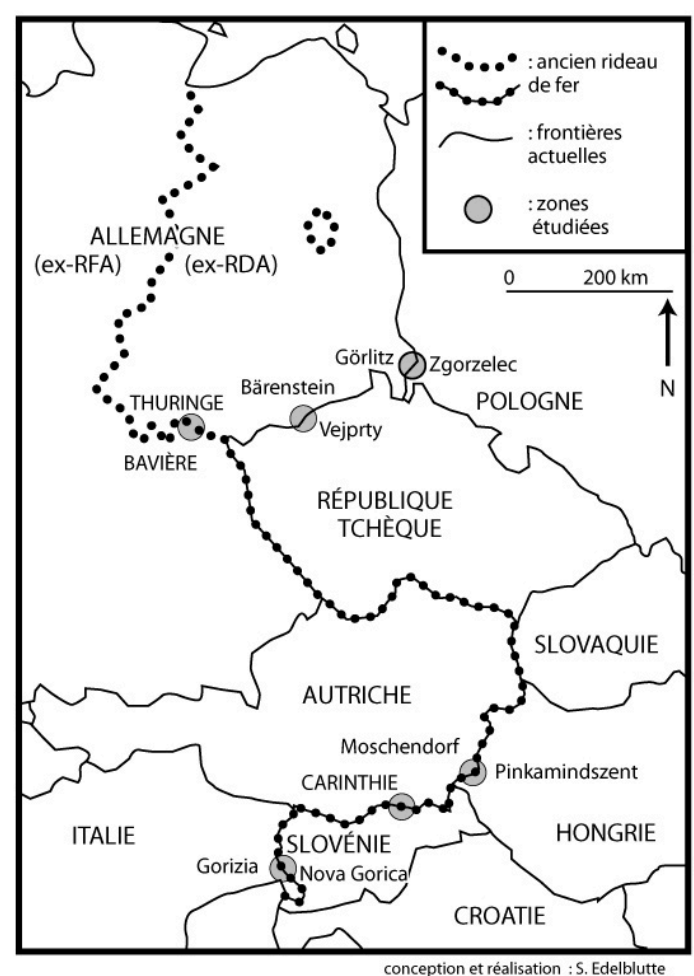

Avec à peine 3000 habitants, la petite commune de Bärenstein possédait jusqu'en 1990 un peu d'industrie manufacturière. Vejprty/Weipert par contre était, jusqu'à la seconde guerre mondiale, une ville fortement industrialisée de plus de 13000 habitants, qui n'abrite plus actuellement que quelques entreprises fournissant de l'emploi pour 3300 habitants. Après la guerre, l'emploi à Bärenstein a été d'abord dominé par l'exploitation de l'uranium par les autorités d'occupation soviétiques, puis par quelques petites entreprises industrielles. Après le tournant de 1989, l'essor espéré par le tourisme a fait long feu, si bien qu'en raison de ses problèmes structurels la région doit lutter contre un fort chômage et une émigration importante, typique des nouveaux Länder. A Vejprty, après l'expulsion de la majorité de la population allemande à la fin de la seconde guerre mondiale, on a installé de nombreux citoyens tchécoslovaques originaires de l'intérieur du pays ou même d'autres régions frontalières. De nouveaux groupes de population ont ainsi immigré dans la région. Du côté saxon, un camp de transit pour les Allemands originaires de l'ancienne Union soviétique (Spätaussiedler) a été implanté il y a quelques années. Du côté tchèque, des Roms se sont installés après la guerre. Enfin, après 1990, il y a eu un afflux accru de marchands vietnamiens à Vejprty. L'article montre les conditions géographiques et démographiques à la frontière germano-tchèque, ainsi que quelques uns des problèmes typiques au regard de l'identité régionale transfrontalière.

Doris Wastl-Walter et Monika Maria Váradi montrent de la même façon la situation dans de petites communes situées à la frontière austro-hongroise. A la différence des deux précédents articles, la situation à cette frontière n'est pas marquée par l'histoire et il n'y a pas eu d'expulsion ni d'échange de population. Aussi, il y aurait des conditions favorables pour une coopération transfrontalière sans problèmes. Jusqu'en 1948, les deux communes étudiées de Moschendorf et de Pinkamindszent étaient 
reliées par différentes sortes de contacts. Avec la construction du rideau de fer, la frontière linguistique et étatique très perméable devint une ligne de séparation entre deux systèmes. Du côté autrichien, on se trouvait dans une région périphérique, économiquement désavantagée, alors que du côté hongrois on était en plus isolé du reste du pays par une frontière intérieure. Après l'ouverture de la frontière en 1989, la grande curiosité initiale s'est transformée en peu de temps en désintérêt et même en absence totale de contact transfrontalier. Depuis l'entrée de l'Autriche dans l'Union européenne en 1995, seuls les hommes politiques locaux essayent de construire une coopération transfrontalière à l'aide de différents programmes de développement européens. Au cours de l'élargissement de l'Union européenne, on a mis l'accent sur les projets qui doivent garantir la transition la plus douce possible vers une vie en commun dans la région frontalière. Aussi bien du côté autrichien que du côté hongrois on travaille sur des concepts de développement transfrontalier qui devraient déboucher sur des projets communs. Mais on retrouve ici le même phénomène que celui décrit par A. Galasinska dans sa contribution : les hommes politiques sont très intéressés par une coopération qui est aussi fortement soutenue par l'Union européenne, mais la population locale est peu intéressée. L'objectif premier de la politique doit d'abord être de surmonter les peurs des citoyens des deux côtés de la frontière et de leur faire saisir concrètement les avantages d'une coopération. Pour le moment, la population est encore très loin de s'identifier au voisin.

Brigitta Busch et Heike Schiebeck s'intéressent de nouveau à une frontière marquée par l'histoire, celle entre l'Autriche et la Slovénie, plus exactement entre la Carinthie et la Slovénie. Cet espace frontalier a été âprement disputé lors des deux guerres mondiales ; de plus, une forte minorité slovène y vit et forme une majorité dans beaucoup de villages. D'autre part, il n'y a jamais eu entre l'Autriche et la Yougoslavie, une frontière aussi hermétiquement close que celle examinée dans les articles précédents et il y a toujours eu une communication et une interaction à travers la frontière. Dans cette contribution, on présente d'abord la situation spécifique à cette frontière, ainsi que les représentations que les habitants se font d'eux-mêmes et de l'étranger. Puis on montre comment, à travers certains projets, on doit font d'eux-mêmes et de l'étranger. Puis on montre comment, à travers certains projets, on doit reconstruire progressivement une coopération transfrontalière plus étroite.

15 Avec son équipe, Emidio Sussi étudie à la frontière italiano-slovène une situation semblable à celle de la frontière germano-polonaise. Là aussi la ville historique, Gorizia, se situe à l'ouest, tandis que Nova Gorica est une ville nouvelle. Cependant, comme dans l'exemple précédent, il n'y avait pas entre l'Italie et la Yougoslavie ou la Slovénie de rideau de fer hermétique. Les dernières décennies ont été pourtant très différemment vécues et les récits des gens font référence d'une part à mille ans d'une histoire commune, d'autre part à la séparation forcée d'après la seconde guerre mondiale. Cela a eu des conséquences particulières pour la population slovène qui s'est retrouvée arbitrairement séparée de part et d'autres de la frontière; cela a eu aussi des conséquences d'un point de vue politique, administratif et économique pour les deux régions frontalières. Avec l'entrée de la Slovénie dans l'Union européenne, le cadre général a changé et on va voir si les deux villes de l'agglomération pourront de nouveau rapidement s'intégrer.

16 Ces travaux empiriques montrent que l'établissement des frontières du siècle passé a eu des effets durables dans la tête des gens. Toutes les études de cas présentent des points 
communs et des spécificités. Mais les résultats montrent que, même là où les gens n'ont pas eu à souffrir de leurs voisins, dans la vie quotidienne on ne fait pas d'effort pour passer les frontières politiques devenues maintenant perméables. Il va falloir déployer de grands efforts pour rendre perceptibles aux citoyennes et aux citoyens les points communs dans la nouvelle Europe et forger ainsi progressivement une identité européenne. Pour le moment, il n'existe pratiquement pas d'identités transnationales, en particulier dans les régions frontalières.

\section{BIBLIOGRAPHIE}

EIKI Berg and VAN HOUTUM Henk (eds) (2003). - Routing Borders Between Territories, Discourses and Practices. Ashgate, Aldershot.

MEINHOF Ulrike (ed.) (2002). - Living (with) Borders Identity Discourses on East-West Borders in Europe. « Border Regions Series ». Ashgate, Aldershot.

Meinhof Ulrike (ed.) (2003). - « Bordering European Identities. Special Issue on Borders ». Journal of Ethnic and Migration Studies, September, Vol. 29, No. 5.

MEINHOF Ulrike and GALASINSKI Darius (2000). - « Photography, memory, and the construction of identities on the former East-West German border ». Discourse Studies, Vol. 2:3 Sage.

\section{AUTEURS}

\section{DORIS WASTL-WALTER}

Geographisches Institut der Universität Bern, Hallerstraße 12, CH-3012 Bern dwastl@giub.unibe.ch

\section{ULRIKE H. MEINHOF}

Chair of German and Cultural studies School of Modern Languages University of Southampton Southampton5017 1BJ, UK - U.H.Meinhof@soton.ac.uk 